\title{
Hypermethylation of Promoter Region of LATS1 - a CDK Interacting Protein in Oral Squamous Cell Carcinomas - a Pilot Study in India
}

\author{
Vijaya Ramakrishna Reddy ${ }^{1}$, Thangavelu Annamalai ${ }^{1}$, Vivek Narayanan ${ }^{2}$, Arvind \\ Ramanathan $^{3 *}$
}

\begin{abstract}
Background: Epigenetic silencing of tumor suppressor genes due to promoter hypermethylation is one of the frequent mechanisms observed in cancers. Hypermethylation of several tumor suppressor genes involved in cell cycle regulation has been reported in many types of tumors including oral squamous cell carcinomas. LATS1 (Large Tumor Suppressor, isoform 1) is a novel tumor suppressor gene that regulates cell cycle progression by forming complexes with the cyclin dependent kinase, CDK1. Promoter hypermethylation of the LATS1 gene has been observed in several carcinomas and also has been linked with prognosis. However, the methylation status of LATS1 in oral squamous cell carcinomas is not known. As oral cancer is one of the most prevalent forms of cancer in India, the present study was designed to investigate the methylation status of LATS1 promoter and associate it with histopathological findings in order to determine any associations of the genetic status with stage of differentiation. Materials and Methods: Tumor chromosomal DNA isolated from biopsy tissues of thirteen oral squamous cell carcinoma biopsy tissues were subjected to digestion with methylation sensitive HpaII enzyme followed by amplification with primers flanking CCGG motifs in promoter region of LATS1 gene. The PCR amplicons were subsequently subjected to agarose gel electrophoresis along with undigested amplification control. Results: HpaII enzyme based methylation sensitive PCR identified LATS1 promoter hypermethylation in seven out of thirteen oral squamous cell carcinoma samples. Conclusions: The identification of LATS1 promoter hypermethylation in seven oral squamous cell carcinoma samples (54\%), which included one sample with epithelial dysplasia, two early invasive and one moderately differentiated lesions indicates that the hypermethylation of this gene may be one of the early event during carcinogenesis. To the best of our knowledge, this is the first study to have explored and identified positive association between LATS1 promoter hypermethylation with histopathological features in oral squamous cell carcinomas.
\end{abstract}

Keywords: LATS1 expression in oral carcinoma - p53 associated protein in oral carcinoma

Asian Pac J Cancer Prev, 16 (4), 1599-1603

\section{Introduction}

Genes that act to suppress the cell division are called "tumor suppressors" because the loss of function of such genes results in uncontrolled proliferation of cells leading to tumorigenesis. One of the best known examples for tumor suppressor gene is the p53 gene (Sharma et al., 2014). In the presence of DNA damaging agents (like toxic chemicals present in tobacco and related products for example that can induce mutation) cellular tumor suppressor proteins block cyclins A, E and D (Lin et al., 2014), and cyclin dependent kinases (CDK) 2, 4 and 6 to arrest the cell cycle (Cooks et al., 2014; Gao et al., 2014). Loss of function of tumor suppressor genes due to mutation(s) or loss of expression of tumor suppressor genes due to epigenetic modification causes the cell cycle machinery to undergo uncontrolled proliferation even in the presence of DNA damaging agents resulting in carcinogenesis (Yang et al., 2010; Liu et al., 2011; Adduri et al.,2014). Several studies, both in vitro and in vivo, have clearly associated mutations and epigenetic modification in tumor suppressor genes with transformation potential, progression and metastasis (Aylon et al., 2011; Rivlin et al., 2011; Mak et al., 2014).

Promoter hypermethylation is one of the most common forms of epigenetic silencing of tumor suppressor genes and is believed to be more frequently involved in cancerous lesions than mutations (Chan et al., 2008). Hypermethylation of several tumor suppressor genes involved in cell cycle regulation such as $p 16^{I N K 4 a}$, p15 ${ }^{I N K 4 b}$,

${ }^{1}$ Oral and Maxillofacial Surgery, Rajah Muthiah Dental College and Hospital, Annamalai University, Annamalai Nagar, ${ }^{2}$ Oral and Maxillofacial Surgery, SRM Kattankulathur Dental College \& Hospital, Potheri, ${ }^{3}$ Enable Biolabs, 37B, Madurai Meenakshipuram Extension, Iyyanchery Main Road, Urappakkam, Chennai, India*For correspondence: arvind@ebl.org.in 
p14 ${ }^{A R F}$, p73, APC and BRCA1 have been reported in many types of tumors (Asokan et al., 2014; Kanherkar et al., 2014), albeit with variations in percent prevalence amongst them (Christoph et al., 2007; Moran et al., 2012). The percent occurrence of hypermethylation, hence, provides a tumor specific gene signature that may be used for sensitive detection of grade and stage of tumor types (Moran et al., 2012). For example, hypermethylation profile of $M G M T$, a gene involved in DNA repair has proven its usefulness in predicting the clinical prognosis and response to therapy in malignant glioma, gastric cancer, and lung cancer (Vallot et al., 2011).

The gene LATS1 (Large Tumor Suppressor, isoform 1) is a novel tumor suppressor molecule, the protein product of which localizes to the mitotic apparatus of a dividing cell (Hao et al., 2009). LATS1 protein forms complexes with the cyclin dependent kinase-CDK1, essential for the cells to progress from $\mathrm{G} 2$ phase to mitotic phase. Binding of LATS1 with CDK1 reduces the activity of CDK1 and thus blocks the cells from entering mitosis (Bothos et al., 2005). Since LATS1 negatively regulates cell division, it is called as a tumor suppressor. Mice that lacks LATS1 gene develop soft-tissue sarcomas and ovarian stromal cell tumors besides being highly sensitivity to carcinogenic agents (Bothos et al., 2005). Hypermethylation and downregulation of LATS1 gene promoter has been reported in breast cancers $(56.7 \%)$ (Takahashi et al., 2005), astrocytomas (63.6\%) (Jiang et al., 2006), myxoid liposarcomas, leiomyosarcomas, and malignant fibrous histiocytomas (Hisaoka et al., 2002; Visser S et al., 2010). Although oral squamous cell carcinoma (OSCC) is one of the most prevalent forms of cancer in India (Mishra et al., 2014) among all reported cancers, the methylation status of LATS1 gene promoter has not been explored. Hence, we decided to investigate the methylation status of LATS1 to understand: $a$ ) its prevalence, and $b$ ) its association with histopathological differentiation.

\section{Materials and Methods}

\section{Patient samples}

Thirteen patients with oral squamous cell carcinoma with confirmed histopathological diagnosis were included in the study after obtaining informed consent from patients. Biopsy tissues were collected in $2 \mathrm{ml}$ microfuge tubes containing $1.5 \mathrm{ml}$ of RNA Save reagent and transferred to the refrigerator. $24 \mathrm{~h}$ later the samples were transferred to freezer and stored until transported to the laboratory.

\section{DNA extraction and methylation sensitive restriction digestion}

Tissues were washed once with cold $1 \mathrm{X}$ PBS (Phosphate Buffered Saline) to remove traces of RNA Save (Biological Industries Israel Beit-Haemek Ltd. Kibbutz Beit-Haemek, 25115, Israel) and were lysed in $500 \mu 1$ of lysis buffer to extract genomic DNA as described elsewhere (Mehta, 2014). 250ng of genomic DNA was digested with methylation sensitive type II restriction endonuclease, HpaII (New England Biolabs, Ipswich, MA, USA). The digestion was performed with $10 \mathrm{U}$ of enzyme in a $20 \mu \mathrm{l}$ reaction volume at $37^{\circ} \mathrm{C}$ for $4 \mathrm{~h}$, after which the enzyme was inactivated by incubating the reaction mix at $65^{\circ} \mathrm{C}$ for $20 \mathrm{~m}$. Methylated DNA (EpiTect Control DNA, cat \# 59655, Qiagen) was included as positive control for enzyme digestion.

\section{Methylation sensitive polymerase chain reaction (MS-} PCR)

The Hpa II digested tumor DNA samples were amplified with a set of LATS1 promoter specific primers. Primers were designed flanking 500bp of promoter sequence upstream of translational start site, since this region is rich in CCGG content (Figure 1). The following set of primer sequences was used: Left primer: AAGTAAAACCTCTTGTCACACC, Right primer: CAATCCTTTTCCCCATTG. PCR was performed in triplicates with the above LATS1 promoter set of primers on HpaII digested or undigested tumor samples and control DNA. Control DNA was obtained from peripheral blood of a healthy volunteer. The following conditions were used: initial denaturation at $94^{\circ} \mathrm{C}$ for 5 minutes, followed by 35 cycles of denaturation at $94^{\circ} \mathrm{C}$ for 45 seconds, primer annealing at $55^{\circ} \mathrm{C}$ for 45 seconds, primer extension at $72^{\circ} \mathrm{C}$ for 45 seconds, with a final extension at $72^{\circ} \mathrm{C}$ for 5 minutes.

\section{Results}

\section{Promoter analysis and primer designing}

The sequence of promoter region of LATS1 was acquired from the public database (www.ncbi.nlm.nih. gov/gene), and 1000 base pairs upstream of transcription start site was analyzed for the relative presence of $\mathrm{CG}$ (Cytosine and Guanine) (Figure 1). A total of fifty six $\mathrm{CG}$ motifs were identified, of which six were within the CCGG tetranucleotide recognition motif of HpaII, type 2 restriction enzyme. Based on the location of the CCGG motifs, forward and reverse primers were designed to encompass the first three CCGG motifs upstream of the transcriptional start site.

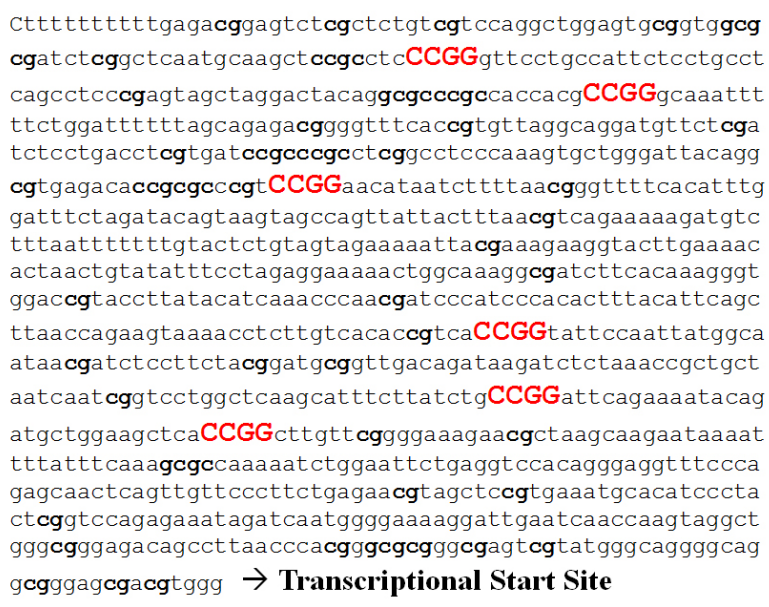

Ctttttttttgagacggagtctcgctctgtcgtccaggctggagtgcggtggcg cgatctcggctcaatgcaagct ccgcct CCCGGgttcctgccattctcctgcct cagcctcccgagtagctaggactacaggcgcccgccaccacgCCGGgcaaattt ttctggattttttagcagagacggggtttcaccgtgttaggcaggatgttctcoa tctcctgacctcgtgatccgccegcctcggcctcccaaagtgctgggattacagg cgtgagacaccgcgeccgtCCGGaacataatcttttaacgggttttcacatttg gatttctagatacagtaagtagccagttattactttaacgtcagaaaagatgtc tttaattttttgtactctgtagtagaaaattacgaaagaaggtacttgaaaac actaactgtatatttcctagaggaaaactggcaaaggcgatcttcacaaagggt ggaccgtaccttatacatcaaacccaacgatcccatcccacactttacattcagc ttaaccagaagtaaaa cctcttgtcacaccgtcaCCGGtattccaattatggca ataacgatctccttctacggatgcggttgacagataagatctctaaaccgctgct aatcaatcggtcctggctcaagcatttcttatctgCCGGattcagaaaatacag atgctggaagctcaCCGG tttatttcaaagcgccaaaatctggaattctgaggtccacagggaggtttccca gagcaactcagttgttcccttctgagaacgtagctccgtgaaatgcacatcccta ctcggtccagagaaatagatcaatggggaaaaggattgaatcaaccaagtaggct gggcgggaga cagccttaacccacgggcgegggcgagtcgtatgggcaggggcag gcgggagcgacgtggg $\rightarrow$ Transcriptional Start Site

Figure 1. DNA Sequence of LATS1 Promoter Region (-1000bp) Upstream of Transcriptional Start Site is Shown. All CG motifs are highlighted in bold letters. CG motifs within the HpaII enzyme recognition site CCGG is highlighted in red 
Table 1. Consolidate Data of Hypermethylation Status and Histopathological Feature of the OSCC Patients

\begin{tabular}{|c|c|c|c|c|}
\hline $\begin{array}{l}\text { Total No. of } \\
\text { Samples }\end{array}$ & $\begin{array}{l}\text { Age and } \\
\text { Gender }\end{array}$ & Location of Tumour & Histo-Pathological Diagnosis & Methylation Status \\
\hline 1 & 60 / Male & Right maxillary alveolus & Early invasive & Methylated \\
\hline 2 & 62 / Female & Left buccal vestibule & Epithelial dysplasia & Methylated \\
\hline 3 & 70 / Male & Right mandibular alveolus & Early invasive & Not methylated \\
\hline 4 & 55 / Male & Right buccal mucosa & Early invasive & Methylated \\
\hline 5 & 65 / Male & Left buccal mucosa & Moderately differentiated & Methylated \\
\hline 6 & 46 / Male & Left buccal mucosa & Moderately differentiated & Not methylated \\
\hline 7 & 42 / Male & Right maxillary alveolus & Moderately differentiated & Not methylated \\
\hline 8 & 65 / Female & Right buccal mucosa & Well differentiated & Not methylated \\
\hline 9 & 45 / Female & Right buccal mucosa & Well differentiated & Methylated \\
\hline 10 & 65 / Male & Left buccal mucosa & Well differentiated & Methylated \\
\hline 11 & 43 / Male & Right buccal mucosa & Well differentiated & Not methylated \\
\hline 12 & 45 / Male & Right buccal mucosa & Well differentiated & Not methylated \\
\hline 13 & 45 / Female & Left mandibular alveolus & Well differentiated & Methylated \\
\hline
\end{tabular}

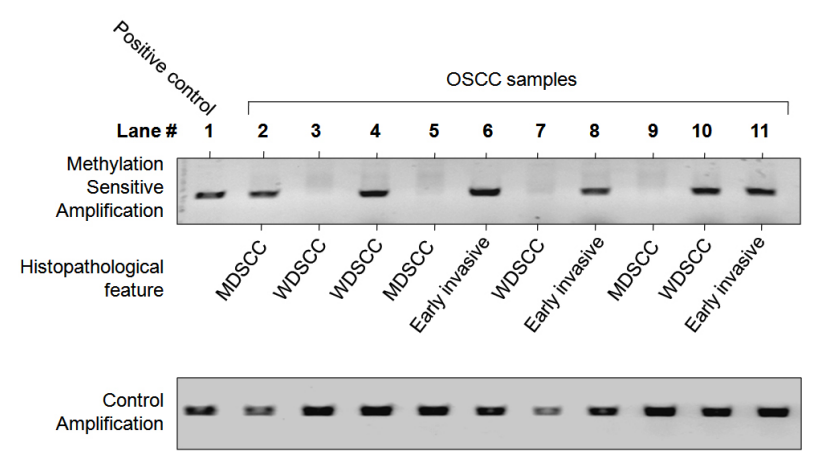

Figure 2.Agarose Gel Electrophoresis of HpaII Enzyme Based Methylation Sensitive PCR. Representative samples are shown. Top panel: PCR amplified products of LATS1 promoter region. Note that only methylated promoters show amplification. Bottom panel: $\mathrm{PCR}$ amplified products of undigested OSCC and control DNA samples. MDSCC: Moderately differentiated oral squamous cell carcinoma. WDSCC: Well differentiated oral squamous cell carcinoma

Restriction digestion of genomic DNA with Hpa II and Polymerase chain reaction to detect hypermethylation of LATS1 promoter region

250 nanograms of genomic DNA from each of the sample were subjected to Hpa II digestion. Hpa II recognizes unmethylated tetranucleotide motif CCGG and cuts after the first $\mathrm{C}$. Incubating the genomic DNA with Hpa II results in complete digestion of all unmethylated CCGG motifs in the entire genome including the promoter region of LATS1, which has six CCGG motifs. The fragmented promoter of LATS1 cannot be amplified by the flanking primers, as the primers were designed to amplify the intact promoter region only. In case, if the $\mathrm{C}$ residue in CCGG motif were to be methylated the Hpa II enzyme would fail to recognize the CCGG motif, which as a result maintains the promoter region intact (uncut). This unfragmented and intact promoter region serves as an effective template and hence the promoter region gets amplified in PCR.

Following digestion of OSCC DNA samples with HpaII, 25 nanogram aliquots of the samples were used as template to amplify the promoter region. Polymerase chain reaction (PCR) was performed in triplicates under conditions mentioned in the methods section. Gel analysis of the PCR product showed amplification in 7 out of $13(54 \%)$ tumor DNA samples, which indicated hypermethylation of the promoter region (Figure 2, top panel, lanes $2,4,6,8,10,11)$ in these samples. The specificity of LATS1 promoter amplification was confirmed by migration of the DNA bands in agarose gel with reference to positive control. Undigested OSCC samples were amplified along with positive control DNA under similar condition as control for the presence of DNA in all the samples, which showed amplification in all tumor DNA samples (Figure 2, bottom panel). Similar data was obtained in the rest of triplicate PCR amplifications (data not shown).

\section{Consolidated data of hypermethylation of LATS1 promoter and histopathology}

All tissue samples that were used in the present study were subjected to histopathological analysis and their diagnosis confirmed independently. Of the thirteen OSCC samples that were analyzed, six were well differentiated, three were moderately differentiated and the remaining four were early invasive lesions. When the hypermethylation status was corroborated with histopathological finding, LATS1 promoter hypermethylation was observed in three of six well differentiated OSCCs, one of three moderately differentiated OSCCs and three of four early invasive lesions (Table 1). No association between the hypermethylation and location of tumor, or age and gender of the patients could be determined due to smaller size of the samples.

\section{Discussion}

In the present study, we have used methylation sensitive restriction enzyme digestion to determine the methylation profile of the promoter region of LATS1, a tumor suppressor gene, in oral squamous cell carcinomas. Our data demonstrates promoter hypermethylation in a significant proportion of cases $(54 \%, n=7$ out of 13 OSCCs). No association between the location of OSCC lesion, age and gender of patients and LATS1 hypermethylation could be made due to smaller size of the samples that were analyzed. The sample size 
was intentionally chosen to include only a few cases in each of the three categories-early invasive, moderately differentiated and well differentiated OSCCs, so as to first explore the occurrence of LATS 1 hypermethylation in OSCC lesions as no prior data on this gene was available in OSCCs. Although bisulfite modification based methylation-specific PCR (MSP) is widely used to determine the hypermethylation status of genes, the assay is difficult to optimize for all loci and requires column based purification procedures and primers for both modified and unmodified DNA. The methylation sensitive restriction enzyme technique, on the other hand, is fast and equally sensitive in detecting the methylation status of genes. This method is also proven to be sensitive in several studies including OSCCs and hence was used in the present study (Bhatia et al., 2014). Besides, the methylation sensitive PCR on HpaII digested DNA was performed in triplicates with similar end results, based on which we believe that the technique is unlikely to have influenced the higher detection of LATS1 hypermethylation in the present study.

The observation of hypermethylation in one lesion with epithelial dysplasia, two early invasive, and one moderately differentiated OSCC samples suggests that the loss of expression of LATS1 may be one of the early events during carcinogenesis. This may be considered as significant as only thirteen OSCC samples were tested altogether. This percent occurrence of LATS1 hypermethylation is similar to the prevalence pattern observed in breast cancers $(56.7 \%)$ (Takahashi et al., 2005) and astrocytomas (63.6\%) (Jiang et al., 2006). The lack of promoter hypermethylation in the rest of the samples indicates that the hypermethylation may not be a targeted event in OSCCs. Such heterogeneity in promoter hypermethylation has also been observed in several genes in several cancers including OSCCs. For example, the cell cycle regulators p15 and p16 are known to be hypermethylated in $23 \%$ of OSCCs, mismatch repair genes- $h M L H 1$ and $M G M T$ are hypermethylated in $8 \%$ and $41 \%$ of OSCCs respectively, while the homotypic cell-cell adhesion gene-E-Cadherin is hypermethylated in $35 \%$ of OSCCs (Marcinkiewicz et al., 2014). The above percent occurrence may be considered as a relevant indicator for heterogeneity of hypermethylation observed in the present study as the authors in the earlier study had analyzed OSCC samples obtained from patients who belonged to the southern region of India from where the samples used in the present study were also collected.

Positive association between promoter hypermethylation and lymph node involvement and metastasis has been observed in other cancers (Peters et al., 2014). In addition, decrease in the levels of LATS1 has also been associated with poor prognosis in breast cancers (Takahashi et al., 2005). This association of LATS1 expression with clinical outcome adds significance to our finding of more frequent hypermethylation in early invasive and moderately differentiated OSCCs than well differentiated OSCCs, as it opens up the possibility of LATS1 methylation status being used as a prognostic indicator and diagnostic biomarker. However, this requires systematic evaluation in a statistically significant number of OSCCs that have been staged and graded. Taken together, the present explorative study has identified for the first time hypermethylation of LATS1 promoter region in histopathologically defined OSCCs.

\section{References}

Adduri RS, Katamoni R, Pandilla R, et al (2014). TP53 Pro72 allele is enriched in oral tongue cancer and frequently mutated in esophageal cancer in India. PLoS One, 9, 114002.

Asokan GS, Jeelani S, Gnanasundaram N (2014). Promoter hypermethylation profile of tumour suppressor genes in oral leukoplakia and oral squamous cell carcinoma. J Clin Diagn Res, 8, 9-12.

Aylon Y, Oren M (2011). New plays in the p53 theater. Curr Opin Genet Dev, 21, 86-92.

Bhatia V, Goel MM, Makker A, et al (2014). Promoter region hypermethylation and mRNA expression of MGMT and p16 genes in tissue and blood samples of human premalignant oral lesions and oral squamous cell carcinoma. Biomed Res Int, 2014, 248419.

Bothos J, Tuttle RL, Ottey M, Luca FC, Halazonetis TD (2005). Human LATS1 is a mitotic exit network kinase. Cancer Res, 65, 6568-75.

Chan TA, Glockner S, Yi JM, et al (2008). Convergence of mutation and epigenetic alterations identifies common genes in cancer that predict poor prognosis. PLoS Med, 5, 823-39.

Christoph F, Hinz S, Kempkensteffen C, et al (2007). A gene expression profile of tumor suppressor genes commonly methylated in bladder cancer. J Cancer Res Clin Oncol, 133, 343-9.

Cooks T, Harris CC (2014). p53 Mutations and InflammationAssociated Cancer Are Linked through TNF Signaling. Mol Cell, 56, 611-2.

Gao SY, Li J, Qu XY, Zhu N, Ji YB (2014). Downregulation of Cdk1 and cyclinB1 expression contributes to oridonininduced cell cycle arrest at $\mathrm{G} 2 / \mathrm{M}$ phase and growth inhibition in SGC-7901 gastric cancer cells. Asian Pac J Cancer Prev, 15, 6437-41.

Hao Y, Chun A, Cheung K, Rashidi B, Yang X (2008). Tumor suppressor LATS1 is a negative regulator of oncogene YAP. J Biol Chem, 283, 5496-509.

Hisaoka M, Tanaka A and Hashimoto H (2002). Molecular alterations of h-warts/LATS1 tumor suppressor in human soft tissue sarcoma. Lab Invest, 82, 1427-35.

Jiang Z, Li X, Hu J, Zhou W, Jiang Y, Li G and Lu D (2006). Promoter hypermethylation-mediated down-regulation of LATS1 and LATS2 in human astrocytoma. Neuroscience Res, 56, 450-58.

Kanherkar RR, Bhatia-Dey N, Makarev E, Csoka AB (2014). Cellular reprogramming for understanding and treating human disease. Front Cell Dev Biol, 12, 67.

Lin H, Fang L, Lin D (2014). Association of cyclin D1 variants with head and neck cancer susceptibility: evidence from a meta-analysis. Asian Pac J Cancer Prev, 15, 5645-51.

Liu H, Xing Y, Yang S, Tian D (2011). Remarkable difference of somatic mutation patterns between oncogenes and tumor suppressor genes. Oncol Rep, 26, 1539-46.

Mak AS (2014). p53 in cell invasion, podosomes, and invadopodia. Cell Adh Migr, 8, 205-14.

McLaughlin SK, Olsen SN, Dake B, et al (2013). The RasGAP gene, RASAL2, is a tumor and metastasis suppressor. Cancer Cell, 24, 365-78.

Mehta DT, Annamalai T, Ramanathan A (2014). Lack of mutations in protein tyrosine kinase domain coding exons 19 and 21 of the EGFR gene in oral squamous cell carcinomas. Asian Pac J Cancer Prev, 15, 4623-7. 
Mishra A, Meherotra R (2014). Head and neck cancer: global burden and regional trends in India. Asian Pac J Cancer Prev, 15, 537-50.

Moran A, Fernandez-Marcelo T, Carro J, et al (2012). Methylation profiling in non-small cell lung cancer: clinical implications. Int J Oncol, 40, 739-46.

Peters I, Dubrowinskaja N, Abbas M, et al (2014). DNA methylation biomarkers predict progression-free and overall survival of metastatic renal cell cancer (mRCC) treated with antiangiogenic therapies. PLoS One, 9, 91440.

Rivlin N, Brosh R, Oren M, Rotter V (2011). Mutations in the p53 tumor suppressor gene: important milestones at the various steps of tumorigenesis. Genes Cancer, 2, 466-74.

Sharma S, Sambyal V, Guleria K, et al (2014). TP53 polymorphisms in sporadic North Indian breast cancer patients. Asian Pac J Cancer Prev, 15, 6871-9

Takahashi Y, Miyoshi Y, Takahata C, et al (2005). Down regulation of LATS1 and LATS2 mRNA expression by promoter hypermethylation and its association with biologicall aggressive phenotype in human breast cancers. Cancer Res, 11, 1380-5.

Vallot C, Stransky N, Bernard-Pierrot I, et al (2011). A novel epigenetic phenotype associated with the most aggressive pathways of bladder tumor progression. J Natl Cancer Inst, 103, $47-60$

Visser S, Yang X (2010). LATS tumor suppressor: a new governor of cellular homeostasis. Cell Cycle, 9, 3892-903.

Marcinkiewicz KM, Gudas LJ (2014). Altered histone mark deposition and DNA methylation at homeobox genes in human oral squamous cell carcinoma. J Cell Physiol, 229, 1405-16.

Yang H, Zhong Y, Peng C, Chen JQ, Tian D (2010). Important role of indels in somatic mutations of human cancer genes. BMC Med Genet, 11, 128. 